\title{
New Fluorinated Terphenyl Isothiocyanate Liquid Crystal Single Compounds and Mixtures
}

\author{
Amanda Parish ${ }^{1}$, Sebastian Gauza ${ }^{1}$, Shin-Tson Wu', \\ Jerzy Dziaduszek ${ }^{2}$, and Roman Dabrowski ${ }^{2}$ \\ ${ }^{1}$ College of Optics and Photonics, University of Central Florida, FL, USA \\ ${ }^{2}$ New Technology and Chemistry Department, Military University of \\ Technology, Warsaw, Poland
}

\begin{abstract}
High birefringence $(\Delta n>0.4)$ nematic liquid crystals are particularly attractive for infrared applications because they enable a thinner cell gap to be used for achieving fast response time. In this paper, we report the mesomorphic and physical properties of several fluorinated NCS terphenyl single compounds and mixtures based solely on laterally fluorinated aromatic rigid core structures. The single compounds have $\Delta n \sim 0.35$ in the visible spectral region and relatively low viscosity. In terms of applications, these compounds can be used as a dopant for enhancing the figure-of-merit of commercial mixtures for display applications. Or we can formulate high birefringence eutectic mixtures using solely NCS terphenyl compounds for near IR laser beam steering applications.
\end{abstract}

Keywords: high birefringence; terphenyl liquid crystals

\section{INTRODUCTION}

High birefringence $(\Delta \mathrm{n})$, low viscosity, and photo- and thermal-stable liquid crystals (LCs) are particularly attractive for infrared applications [1,2], such as laser beam steering at $\lambda=1.55 \mu \mathrm{m}[3]$ and foveated imaging [4]. For these phase modulation applications, a minimum $2 \pi$ optical phase change $(\delta=2 \pi \mathrm{d} \Delta \mathrm{n} / \lambda$, where $\mathrm{d}$ denotes cell gap of the homogeneous LC cell and $\lambda$ is wavelength) is required. To compensate for the longer wavelength in the infrared region, a larger optical path

This work is supported by DARPA Bio-Optics Synthetic Systems program under Contract No. W911NF04C0048, and NATO Programme Security Through Science, Collaborative Linkage Grant No. CBP.EAP.CLG 981323.

Address correspondence to Shin-Tson $\mathrm{Wu}$, College of Optics and Photonics, University of Central Florida, Orlando, FL 32816, USA. E-mail: swu@mail.ucf.edu 
length $(\mathrm{d} \Delta \mathrm{n})$ is needed. To achieve this goal, we could either increase the LC cell gap or increase the LC birefringence. However, increasing cell gap would lead to a sluggish response time, which is proportional to $\mathrm{d}^{2}$ and visco-elastic coefficient $\left(\gamma_{1} / \mathrm{K}_{11}\right)$. To meet the response time requirements, the two preferred approaches are to have low rotational viscosity $\left(\gamma_{1}\right)$ LC mixtures and to reduce the cell gap [5-7]. However, high birefringence and low rotational viscosity are opposing LC characteristics. A highly conjugated LC compound usually exhibits a high viscosity because of its increased moment of inertia. Moreover, high birefringence LC compounds usually possess high melting temperatures. To lower the melting temperature, many LC structures need to be developed and eutectic mixtures formulated.

The most effective method of increasing birefringence is to elongate the $\pi$-electron conjugation of the LC compounds [8-13]. Conjugation length can be extended by either multiple bonds or unsaturated (phenyl) rings in the rigid core structure. However, the following problems are associated with highly conjugated LC compounds: high melting temperature, increased viscosity, reduced UV stability, and relative low resistivity due to ion trapping near the polyimide (PI) alignment interfaces [14]. The high melting temperature can be combated through the lateral fluorination of the rigid core and use of eutectic mixtures. The increased viscosity is inherent to all the highly conjugated compounds but can be mitigated by choice of polar group. UV stability can be improved by forming the rigid core of unsaturated rings instead of multiple bonds, as double and triple bonds are more susceptible to UV absorption [8,15-16]. Recently it was shown that by using a multi-step purification process even a highly polar mixture can achieve resistivity at the level of $10^{-13} \mathrm{ohm} / \mathrm{cm}[17,18]$. For the purpose of elongating molecular conjugation, common rigid cores are phenyl-tolane and terphenyl structures and common polar groups are cyano $(\mathrm{CN})$ and isothiocyanato (NCS). Due to relatively low visco-elastic coefficients and improved photo-stability, NCS terphenyl compounds are preferable to cyano $(\mathrm{CN})$ terphenyl compounds or phenyl-tolane based rigid core compounds. Although the linear structure of cyano terphenyls creates a larger dipole moment $(\mu=4.1 \mathrm{D})$ than NCS terphenyls ( $\mu=3.7 \mathrm{D})$, the strong polarization of the carbon-nitrogen triple bond creates large, highly localized Huckel charges. Consequently, dimers are formed by strong molecular interactions between the nitrile groups and a significant increase in viscosity is observed. In contrast, NCS compounds do not form dimers, and therefore rotational viscosity is significantly lower than analogous CN compounds [19]. Based on the above reasoning, several isothiocyanate terphenyl compounds with different lateral fluorine substitutions were synthesized and studied. 
In this paper molecular structures, mesomorphic properties, and electro-optic properties of six multiple fluorinated isothiocyanatoterphenyl single compounds and mixtures based on these compounds are reported.

\section{EXPERIMENTAL}

Several measurement techniques were utilized to characterize the physical properties - including birefringence, visco-elastic coefficient, and Figure of Merit - of single LC compounds and mixtures in our laboratory. Measuring birefringence greater than 0.3 (at $\lambda=633 \mathrm{~nm}$ ) required electro-optic measurements, as the extraordinary refractive index of the LC under study exceeded the upper limit $\left(n_{e}>1.80\right)$ of the Abbe refractometer used in the classic refractometric method. For the electro-optic method, homogenous cells with cell gaps of $5 \mu \mathrm{m} \pm 0.30 \mu \mathrm{m}$ were filled at elevated temperatures above the melting point of the LC under study. The optical setup included a linearly polarized He-Ne laser $(\lambda=632.8 \mathrm{~nm})$ as the light source, a linear polarizer oriented at $45^{\circ}$ with respect to the LC cell rubbing direction, and an analyzer oriented perpendicular to the polarizer. The light transmittance was measured by a photodiode detector (New Focus Model 2031) and recorded digitally by a LabVIEW data acquisition system (DAQ, PCI 6110). Experimental driving parameters were a $1 \mathrm{kHz}$ square-wave AC signal with peak to peak amplitude ramped from $0 \mathrm{~V}$ to $5 \mathrm{~V}$ that was applied to indium-tin-oxide (ITO) electrodes coated on the inner surfaces of the LC cell. The cell substrates were coated with a thin layer of polyimide alignment film, deposited on top of the ITO electrodes, and buffed to induce a $2^{\circ}-3^{\circ}$ pretilt angle. The cell was mounted in a Linkam LTS 350 Large Area Heating/ Freezing Stage controlled by a Linkam TMS94 Temperature Programmer. The phase retardation $(\delta)$ of the homogeneous cells were then measured by the LabVIEW system. The LC birefringence $(\Delta n)$ at wavelength $\lambda$ and temperature $\mathrm{T}$ was obtained from the phase retardation $(\delta)$ measurements using the following equation [20]:

$$
\delta=2 \pi d \Delta n / \lambda
$$

By measuring the free relaxation (decay) time for a controlled phase change, visco-elastic coefficient $\left(\gamma_{1} / K_{11}\right)$ was calculated according to the following equations [21]:

$$
\begin{gathered}
\delta(t)=\delta_{0} \exp \left(-2 t / \tau_{0}\right) \\
\tau_{0}=\gamma_{1} d^{2} /\left(K_{11} \pi^{2}\right)
\end{gathered}
$$


where $\delta_{0}$ is the total phase change, $\gamma_{1}$ is rotational viscosity, $\mathrm{d}$ is cell gap, and $\mathrm{K}_{11}$ is the splay elastic constant. To compare the performance of LC mixtures, a figure-of-merit (FoM) that takes the phase retardation and visco-elastic coefficient into account was defined as [22]:

$$
\text { FoM }=K_{11}(\Delta n)^{2} / \gamma_{1}
$$

Each of the three physical properties $-\Delta n, \gamma_{1} / K_{11}$, and FoM - was known to be temperature dependent. Therefore, the heating/freezing stage was used to control the environmental conditions such that experimental data could be collected at approximately $5^{\circ} \mathrm{C}$ intervals over the entire nematic range of the LC material under study. Since the single LC compounds under study have nematic phases at high temperatures (above $100^{\circ} \mathrm{C}$ ), fitting parameters are used to extrapolate the physical properties at room temperature.

All the thermal analyses were performed using a high-sensitivity differential scanning calorimeter (DSC, TA Instrument Model Q-100). Phase transition temperatures were measured at a scanning rate of $2{ }^{\circ} \mathrm{C} / \mathrm{min}$.

\section{RESULTS \& DISCUSSION}

This experiment focused on the thermotropic, rod-like molecular systems with a polar isothiocyanate (NCS) terminal group. Several variations of lateral fluorine substitutions were studied, with the goal of achieving the highest $\Delta n$ value as possible while retaining a relatively low viscosity. For this purpose, the rigid core structure of the LC compounds under study was aromatic terphenyl rings, as the unsaturated residues are rich in $\pi$-electrons. These rings are particularly desirable to elongate the $\pi$-electron conjugation thru the rodlike molecule by increasing the polarizability along the principal molecular axis. At the same time photochemical stability should not be jeopardized.

Based on previous experience [23] with highly conjugated linear molecular structures, three different configurations of laterally fluorinated terphenyl isothiocyanates were investigated, with two homologues of each structure. Table 1 lists the compound structures and their mesomorphic properties. For comparison, Figure 1 illustrates phase transition temperatures with respect to the different lateral fluorine substitutions. The clearing points of the double fluorinated structures were found to be the highest (Compounds 5 and 6). Compound 2 showed the highest melting point temperature and lowest clearing point temperature among investigated group of structures. Moreover, heat enthalpy of the melting point of Compound 2 


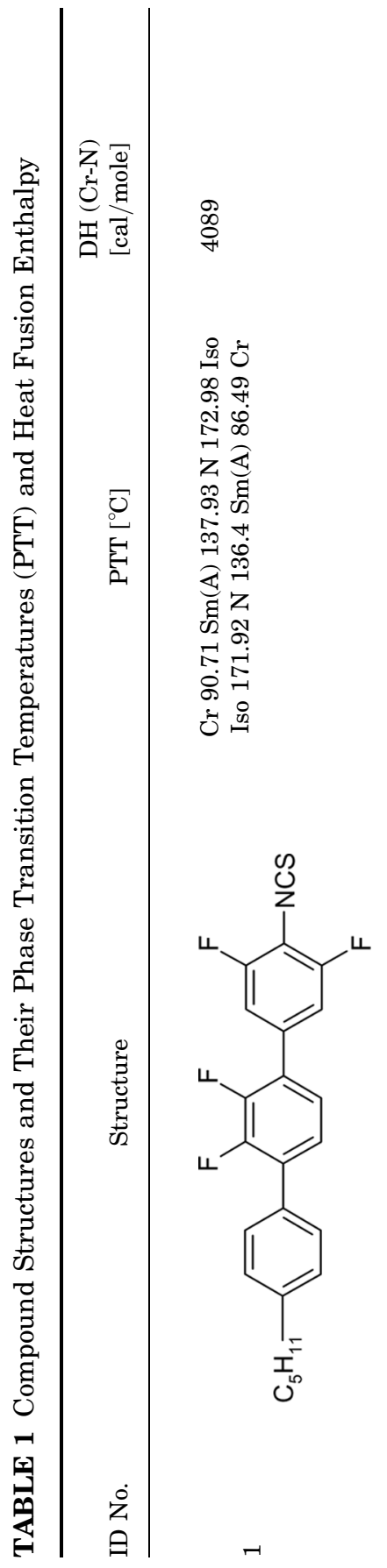

ㅇํㅇ

ญิ

온

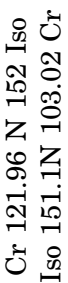

ชู 5

ษึ

茯

ㄱำ

公宫

च

ᄃ

ชำ

రิ
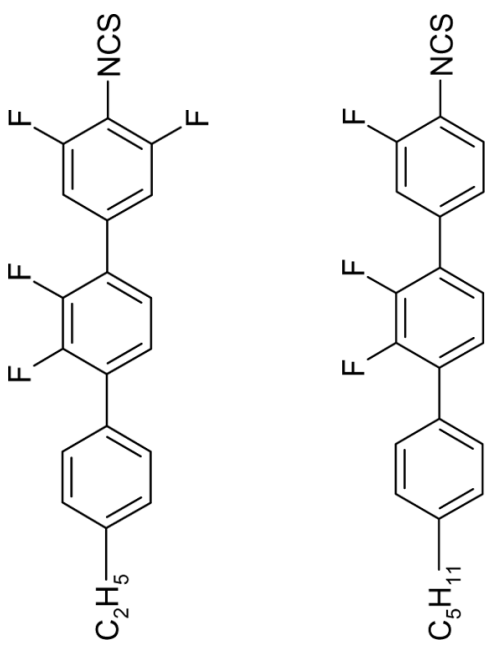

$N$

$\infty$ 
管

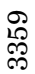

$\stackrel{a}{a}$

远

กิ 10

लृ

๒

ஜ

藏芯

ษึ

낭어

है 乙

ริ งิ่

ठํㅛ

단

คิ่ ㅇํㅇ

乙 乙

$\infty$ o

ํํㅇ 요

రు ఊ

ㅇำ-1

$\infty$ హี

兄岕

금

至든

कू

10

के<smiles>[CH]c1ccc(-c2ccc(-c3ccc([Se][O])cc3)c([AlH2])c2[AlH2])cc1O</smiles>

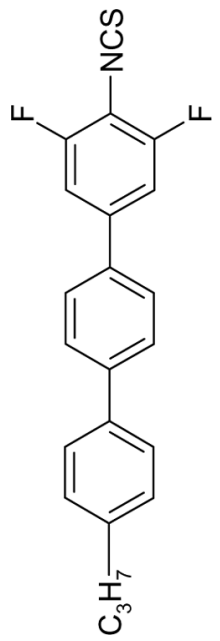<smiles>[CH]c1cc(-c2ccc(-c3ccc([C+]=[O+])cc3)cc2)cc([Hg])c1[O]</smiles>

H

10

6 


\section{Phase Transition Temperatures}

[1] C5PP\{23F\}P\{35F\}-NCS

[2] C2PP(23F)P(35F)-NCS

[3] C5PP(23F) $\{(3 F)-N C S$

[4] $\operatorname{CAPP}(23 F)$ F $\{3 F\}-N C S$

[5] C3PPP(35F)-NCS

[6] C5PPP(35F)-NCS

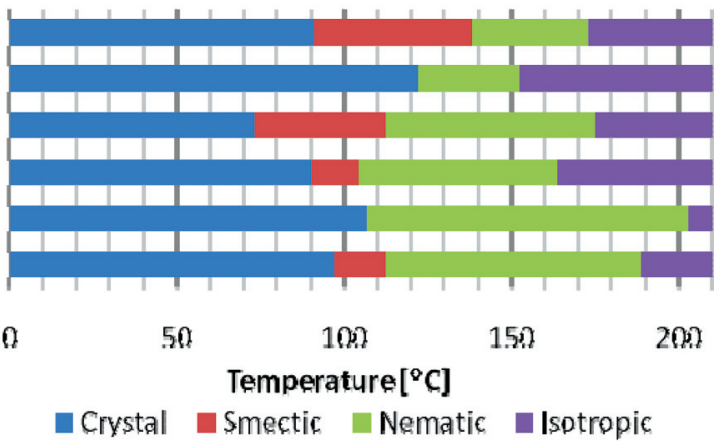

FIGURE 1 Phase Transition Temperatures (PTT) comparison for investigated structures.

exceeded $7 \mathrm{kcal} / \mathrm{mole}$. Such properties suggest that a two carbon chain was too short for long and rigid terphenyl molecular core to provide attractive mesomorphic properties by means of low melting point temperature and wide temperature range of the nematic phase, even if the rigid core was extensively fluorinated. For the melting point temperature comparison, pentyl homologues were chosen. Surprisingly, lateral substitution of four fluorines did not lower the melting point temperature further than triple fluorination. From the mesomorphic properties view point, double fluorination at the phenyl ring with NCS terminal group was the most favorable. The melting point temperatures of the pentyl homologues with different degrees of lateral fluorination follow the order shown below:

$$
\text { Comp. } 3<\text { Comp. } 1<\text { Comp. } 6
$$

As is common with highly conjugated linear structures, smectic phase was observed in four of the six compounds under study. The number of fluorine substitutions had no obvious affect on suppressing the smectic transition. However, a shorter alkyl chain did suppress the smectic phase in both the double and four- fluorinated structures (Comp. 2 and 5). If the smectic to nematic transition was considered, double and triple fluorinated compounds showed similar transition temperatures. Clearing temperature was significantly higher for the double fluorinated compound (Comp. 6):

$$
\text { Comp. } 1 \approx \text { Comp. } 3<\text { Comp. } 6
$$

Shorter chain homologues of double and triple fluorinated terphenyls did not show smectic phase in the phase transitions sequence. We were 
not successful in obtaining less than a four carbon chain homologue of the triple fluorinated isothiocyanato terphenyl with sufficient chemical purity, thus data was not included in this comparison. Further synthesis must be conducted in order to specifically prove whether absence of a smectic phase was an effect of double substitution at $3^{\prime \prime}, 5^{\prime \prime}$ positions of the phenyl ring with polar terminal group or if the length of flexible alkyl chain was the determining factor in suppressing the smectic phase.

Melting heat enthalpy was determined using DSC analysis, and found the double fluorinated compounds to have the lowest $\Delta \mathrm{H}$ values $(<3400 \mathrm{cal} / \mathrm{mole})$, the three-fluorinated compounds to have mid-range $\Delta \mathrm{H}$ values around $4600 \mathrm{cal} / \mathrm{mole}$, and the four-fluorinated compounds to have mid-range (4089 cal/mole for Comp. 1) and high $(7040 \mathrm{cal} / \mathrm{mole}$ for Comp. 2) $\Delta \mathrm{H}$ values. As Figure 1 illustrates, Compounds 5 and 6 are promising candidates for eutectic mixture formulation as they exhibited the best mesomorphic properties: widest nematic range, relatively low melting points, the highest clearing points, low melting heat enthalpy, and no evidence of a smectic phase for Comp. 5.

High birefringence $(\Delta \mathrm{n})$ values were expected for the compounds in question, as the unsaturated rings of the terphenyl structure elongate the $\pi$-electron conjugation thru entire rigid core of the molecules. Laterally unsubstituted terphenyl compounds have been known to exhibit very high melting point temperatures. To lower the melting point temperature and diminish the tendency of terphenyl compounds to form a smectic phase, multiple lateral fluorine substitutions were made. However, this lowers the birefringence of the compound, as laterally substituted fluorine atoms trap $\pi$-electrons and pull them away from the conjugation along the main molecular axis.

The experimental data and fitting results for birefringence of the six compounds are illustrated in Figure 2, and show birefringence values greater than 0.36 for all structures when extrapolated to room temperature $\left(25^{\circ} \mathrm{C}\right)$. Figure 3 illustrates the fitted curves plotted against reduced temperature $(\mathrm{T} / \mathrm{Tc})$ in order to account for the different clearing temperature so that the structural effects can be compared. Of the three differently fluorinated systems studied, homologues C3PPP(3,5F)-NCS and C5PPP(3,5F)-NCS (Compounds 5 and 6) exhibited largest birefringence, 0.4430 and 0.4153 , respectively, when extrapolated to room temperature. This fluorination structure exhibited particularly desirable performance parameters due to the fluorine positions being adjacent to the polar NCS group, as an electron potential is created at one end of the molecule and thereby increases the polarizability of the molecule at the microscopic scale. This, combined with high clearing point temperatures, increases the 


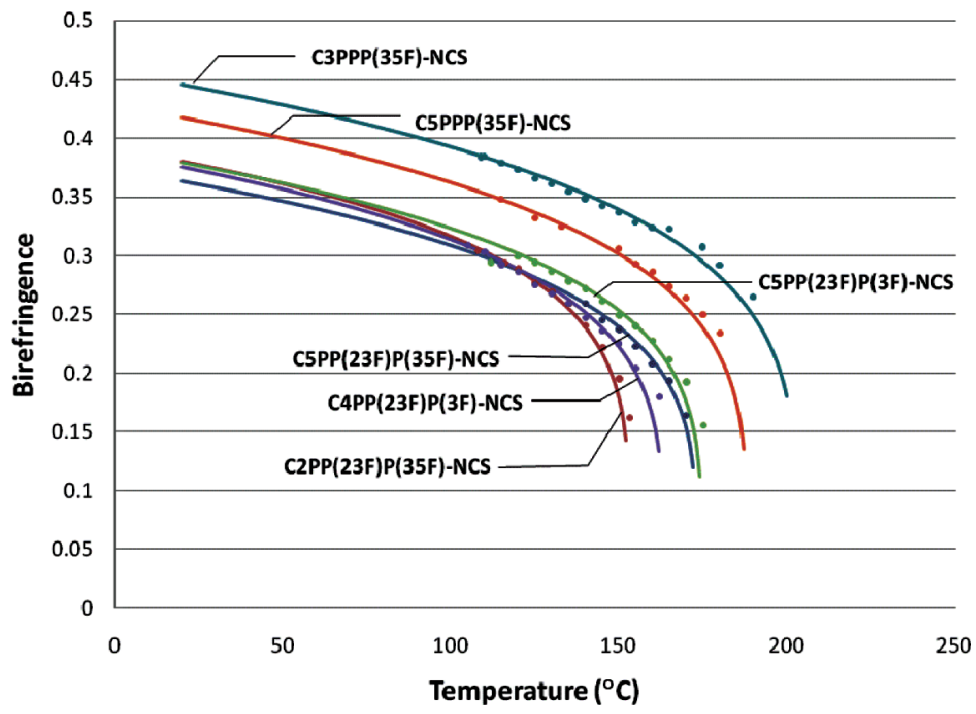

FIGURE 2 Temperature dependent birefringence of the LC compounds studied.

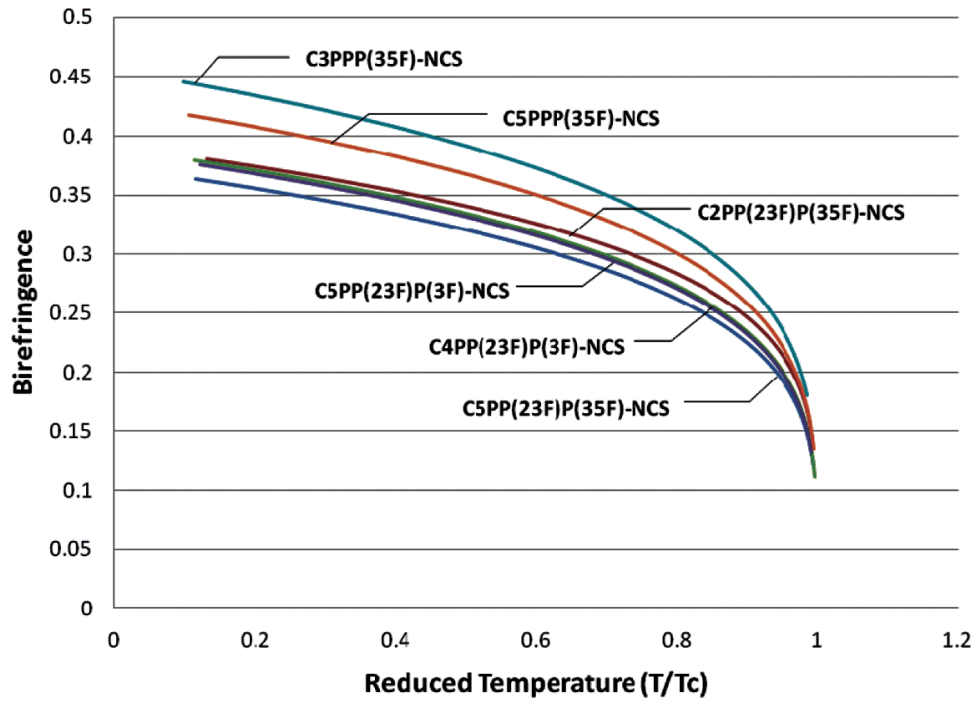

FIGURE 3 Birefringence vs. Reduced Temperature. 
total birefringence while maintaining the suppression of the smectic phase in Compound 5. Introducing a third lateral fluorine and reconfiguring the position of the other two fluorine to $2^{\prime}, 3^{\prime}, 3^{\prime \prime}$ positions of the terphenyl rigid core (4-alkyl-2 $2^{\prime}, 3^{\prime}, 3^{\prime \prime}$-trifluoro- $4^{\prime \prime}$-isothiocyanato$\left[1,1^{\prime} ; 4^{\prime}, 1^{\prime \prime}\right]$ terphenyl, Comp. 3 and 4$)$ decreases birefringence significantly as shown in Figure 2. When the reduced temperature is used to offset the effect of clearing point temperature, loss of birefringence due to additional third fluorine and lateral substituent reconfiguration is more evident over the entire temperature range of the nematic phase. Among investigated compounds, tetra fluorinated terphenyls demonstrate the lowest birefringence with respect to the alkyl chain length which further decreases $\Delta \mathrm{n}$ as the structure is elongated.

The visco-elastic coefficient $\left(\gamma_{1} / K_{11}\right)$ is calculated from the freerelaxation time and characterized the amount of time and energy required to rotate the LC molecules. Figure 4 summarizes both experimental data and fitting results for the six compounds under study and Figure 5 illustrates the fitted curves plotted against reduced temperature (T/Tc). From Figure 5, it is evident that the structures with more fluorine substitutions have higher visco-elastic coefficients over the entire nematic range. This is anticipated, as the structures with more fluorine substitutions have higher molecular weight with

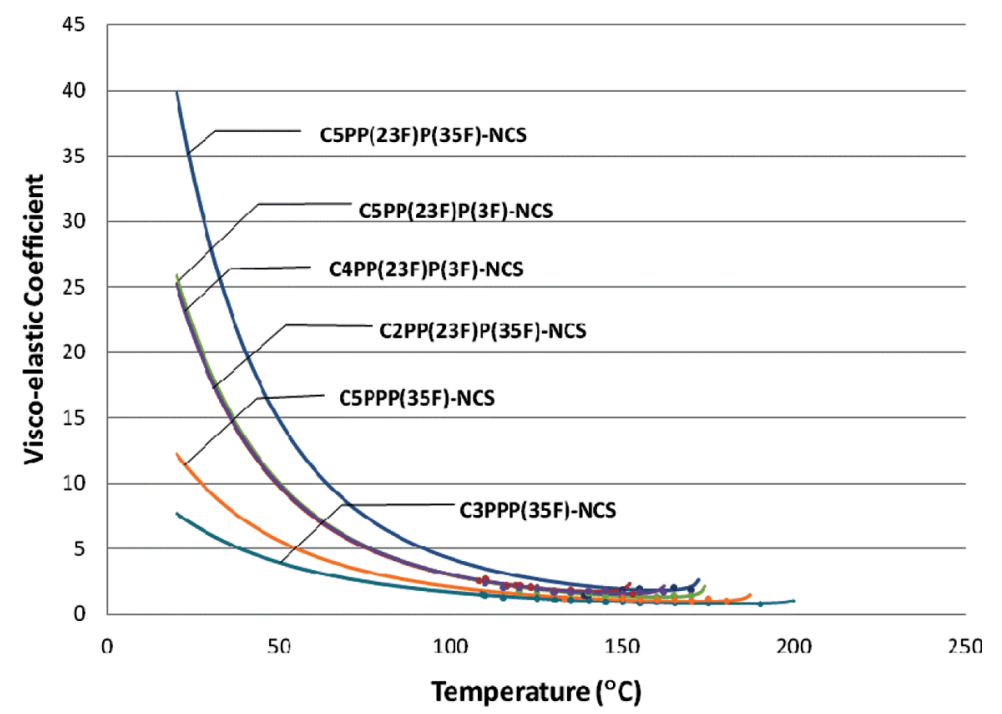

FIGURE 4 Temperature dependent visco-elastic coefficient $\gamma_{1} / \mathrm{K}_{11}$ of the LC compounds studied. 


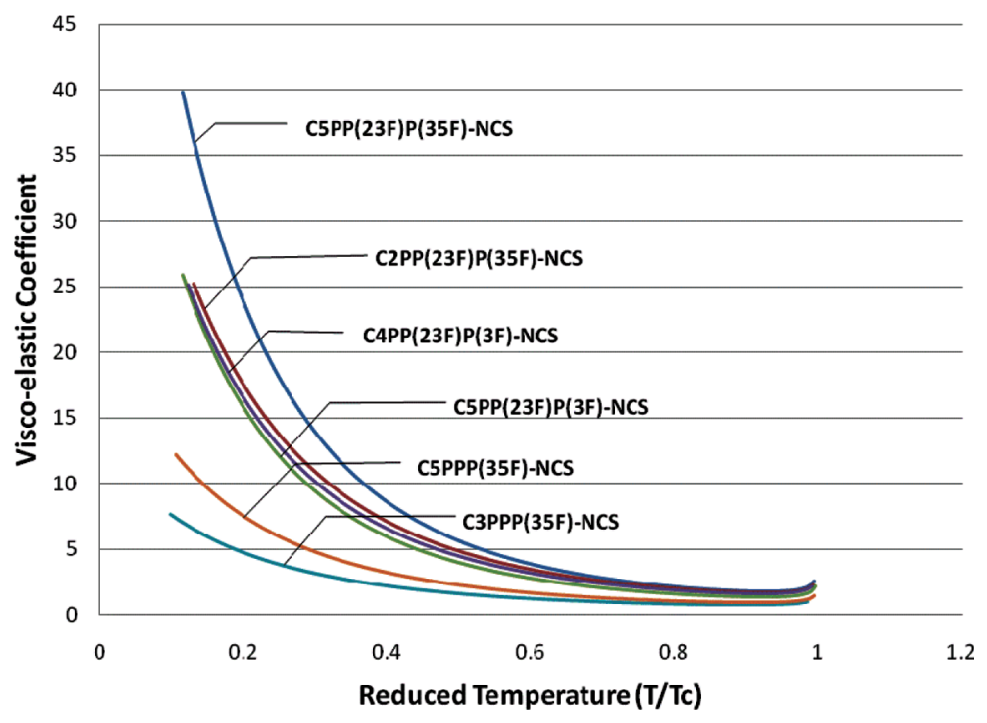

FIGURE $5 \gamma_{1} / K_{11}$ vs. Reduced Temperature.

slightly larger cross-section, and may be a subject for stronger molecular interactions with neighboring molecules due to additional slightly negative charges on the surface. Considering all the above, it can be surmised that additional fluorine substitutions will negatively affect the visco-elastic coefficient. This can be easily analyzed on the basis of Figures 4 and 5.

Figure-of-Merit (FoM) was used for comparison of compound switching speed. Birefringence and visco-elastic coefficient can be compared directly, but response time can only be compared if each compound is studied utilizing a cell gap that exactly matches its birefringence in order to result in the best possible response time. FoM eliminates the necessity of matching cell gap to birefringence, as the parameter is independent of cell gap and allows side-by-side comparison of the different compounds. Moreover, because LC performance and response time is determined by several temperature dependent factors, FoM allows ideal operating temperature to be determined. Also, if an operating temperature is specified - e.g. room temperature - the best compound can be easily determined by the experimental data fitting according to the following equation [22]:

$$
F_{O} M=a(\Delta n)^{2}\left(1-\frac{T}{T_{c}}\right)^{3 \beta} \exp \left(\frac{-E}{\kappa T}\right)
$$


where $a$ is a fitting parameter. Experimental data and appropriate fitting results of the six investigated isothiocyanatoterphenyls are summarized in Figure 6 and fitted curves plotted versus reduced temperature are summarized in Figure 7. It is evident that the double fluorinated terphenyl homologues (Compounds $\mathbf{5}$ and $\mathbf{6}$ ) are superior to trifluorinated or tetrafluorinated compounds, with almost $200 \%$ higher FoM if C3PPP(35F)NCS compound is compared with the others at the optimum operating temperature. The fitted curves show that ideal operating temperature is in the range of $130-160^{\circ} \mathrm{C}$ ( $\mathrm{FoM}$ of 118 and 82 for Comp. 5 and 6 respectively), but that even at an operating temperature of $60^{\circ} \mathrm{C} \mathrm{FoM}$ values remain relatively high (55 and 35 for 5 and 6 respectively). The significantly higher FoM observed of the double fluorinated compounds is due to both higher birefringence and lower visco-elastic coefficient values compared to the structures with more fluorine substitutions. As expected, compounds with higher numbers of laterally substituted fluorine atoms and longer terminal alkyl chain demonstrate lower FoM performance and therefore are expected to be less effective in multi-component compositions intended to reduce response time to applied electric field.

Based on the single compound results, a mixture comprised of Compounds 1, 2, 4, 5, and 6 was formulated (UCF-Base). The mesomorphic properties of this multi-flourinated NCS terphenyl mixture

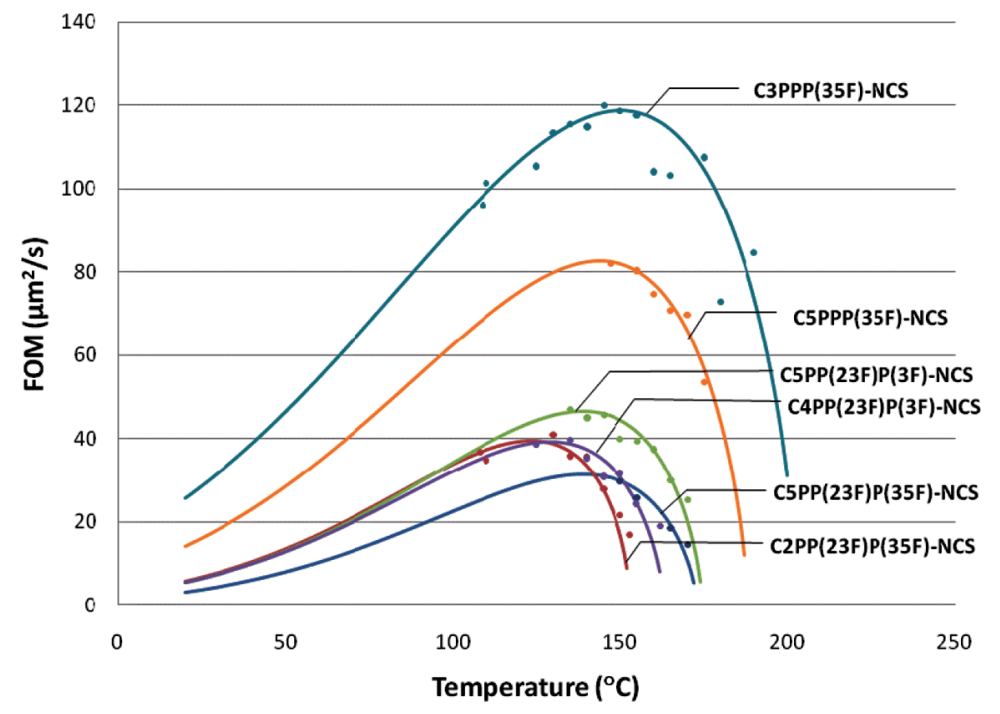

FIGURE 6 Temperature dependent FoM of the LC compounds studied. 


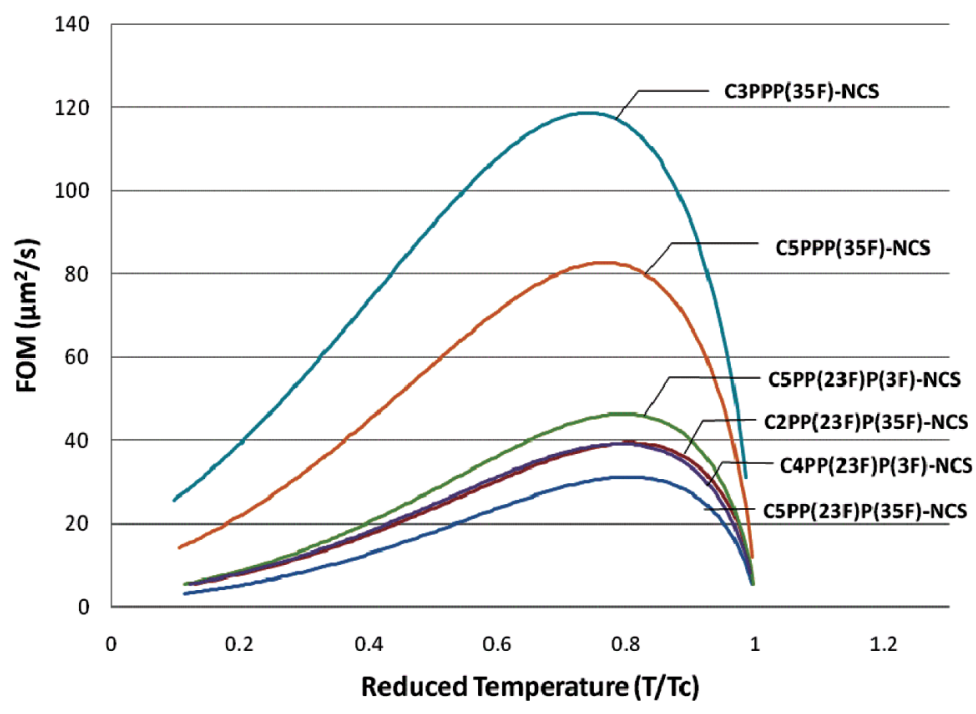

FIGURE 7 FOM vs. Reduced Temperature.

exhibited the melting point at $37.4^{\circ} \mathrm{C}$, the clearing point at $183.6^{\circ} \mathrm{C}$, and a smectic phase extending from the melting point to $114.9^{\circ} \mathrm{C}$, as shown in Fig. 8. The melting point of the mixture was 30 to 80 degrees lower than its constituents and its clearing point remained comparable. The smectic phase of the mixture was unsurprising, as most of the compounds it is comprised of also demonstrated smectic phases. The electro-optic properties UCF-Base demonstrate a very high birefringence, $\Delta \mathrm{n}=0.4$ when extrapolated to $25^{\circ} \mathrm{C}$ (Fig. 9), a relatively low visco-elastic coefficient (Fig. 10), and a Figure of Merit of nearly 70 at the ideal operating temperature of $125^{\circ} \mathrm{C}$ (Fig. 11). However, due to the wide temperature range of a smectic phase this mixture is ill-suited for devices operating at room temperature. Therefore, dopants must be introduced in order to suppress the smectic phase and lower the melting point of the mixture. In order to maintain the high birefringence of the mixture as well as lower the melting point, fluorinated NCS tolanes of the following structure were used:

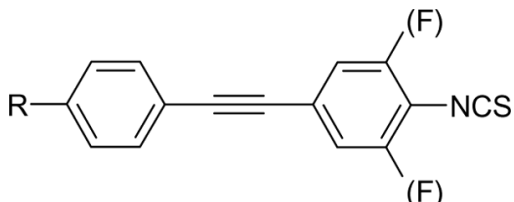

(F)

These compounds were chosen as dopants because they exhibit high birefringence and show no evidence of a smectic phase. Two mixtures 


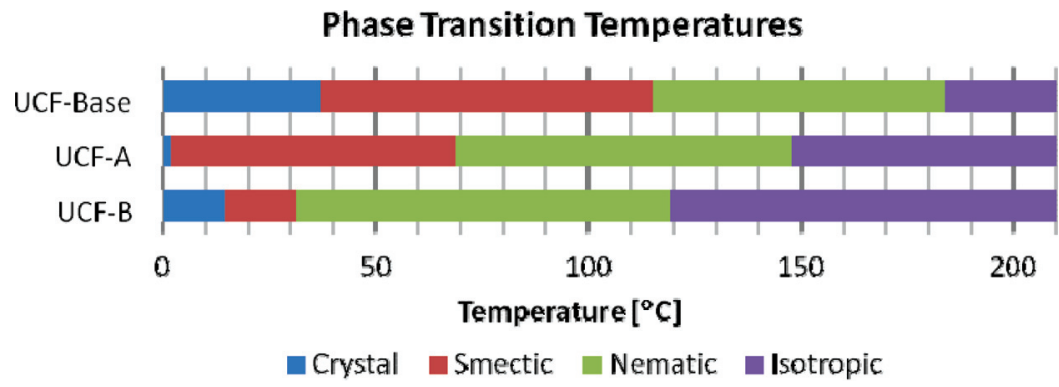

FIGURE 8 Phase Transition Temperatures (PTT) comparison for mixtures.

were designed using the fluorinated NCS terphenyl mixture (UCFBase) as the host and fluorinated NCS tolanes as the guest, one containing $20 \%$ of the guest mixture (UCF-A) and the other containing $40 \%$ (UCF-B). The mesomorphic properties (Fig. 8) of the guest-host systems were significantly improved compared to the host mixture. For the $20 \%$ guest system (UCF-A) the melting point was reduced to $2{ }^{\circ} \mathrm{C}$, the clearing point was $147^{\circ} \mathrm{C}$, and the smectic to nematic transition was lowered by $46^{\circ} \mathrm{C}$ to $69^{\circ} \mathrm{C}$. The $40 \%$ guest system (UCF-B) shows further improvement, exhibiting a melting point of $-4^{\circ} \mathrm{C}$, a clearing point of $119^{\circ} \mathrm{C}$, and the smectic to nematic transition was reduced by nearly 84 degrees. It is clearly evident that the addition of the guest mixture significantly lowered both melting and clearing points, thereby appreciably reducing the operating temperature of

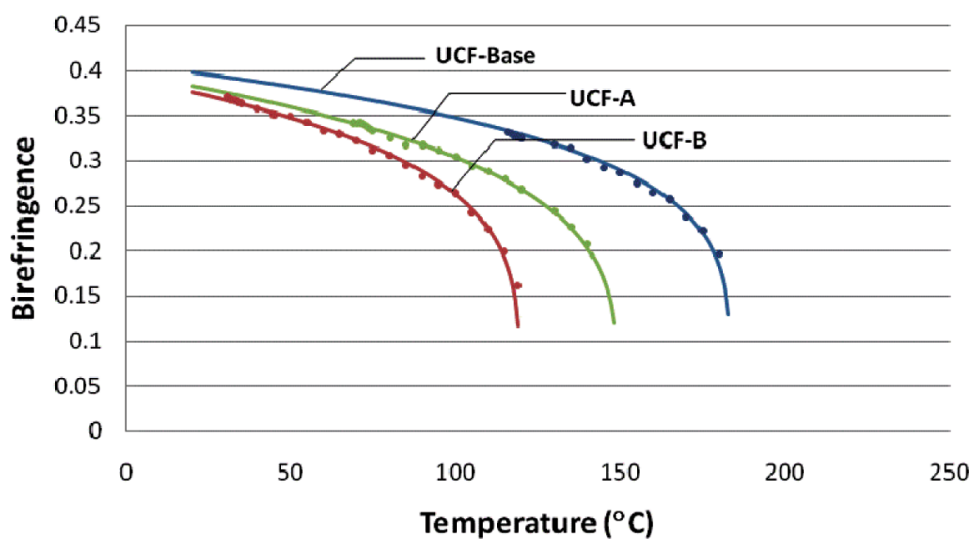

FIGURE 9 Temperature dependent birefringence of LC Mixutres. 
the mixture. The electro-optic properties of the $20 \%$ guest-host system demonstrate a birefringence of 0.38 at $25^{\circ} \mathrm{C}$. Increasing the percentage of the guest mixture causes the birefringence to drop slightly to 0.37 at $25^{\circ} \mathrm{C}$. The lower birefringence of the guest-host systems were primarily due to the lower clearing points of the systems, not because the phenyl-tolane dopants have a birefringence less than the host mixture [11]. The visco-elastic coefficient of the $20 \%$ guest-host system exhibited very little change compared to the UCF-Base host alone. However, further doping the system with $40 \%$ of the guest showed a significant increase in the visco-elastic coefficient (Fig. 10). Despite retaining relatively high birefringence, the Figure of Merit at optimal temperature demonstrated by the guest-host systems was lower than the terphenyl host mixture (UCF-Base) due to lower clearing temperatures and higher visco-elastic coefficients. However, even the lower Figure of Merit demonstrated by UCF-B is higher than the commercially available liquid crystals [24].

The presented results of the multi-fluorinated NCS terphenyl base mixture and subsequent guest-host mixtures demonstrate potential usefulness in liquid crystal devices, such as optical phased arrays. Further investigation of such systems will lead to improved eutectic mixture formulations with lower melting temperatures and will diminish or eliminate smectic phase. The choice of dopant compounds will be the critical factor in designing mixtures with higher birefringence and improved operating temperatures. As demonstrated in the

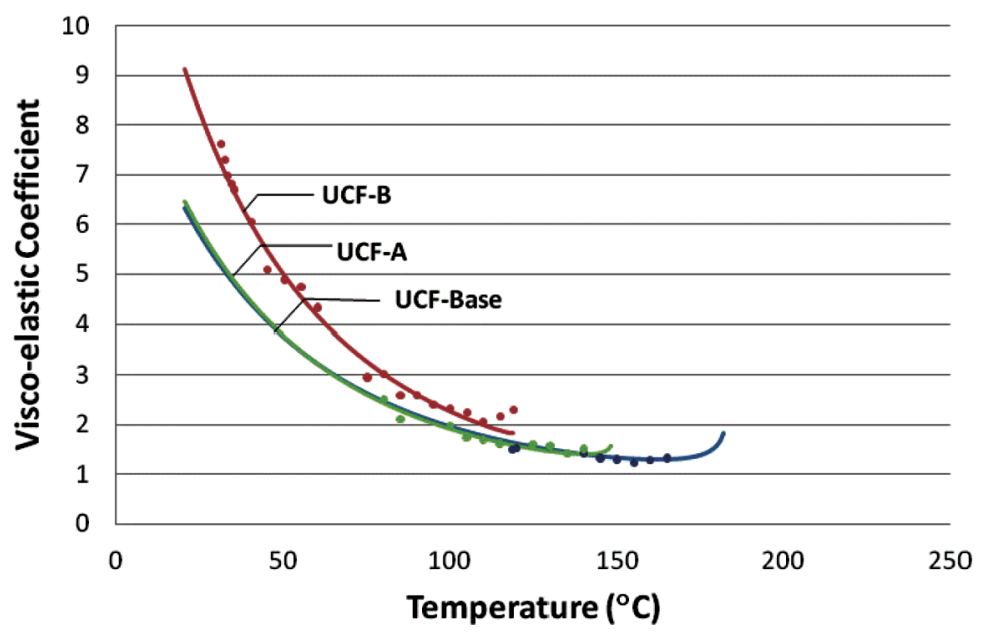

FIGURE 10 Temperature dependent visco-elastic coefficient $\gamma_{1} / \mathrm{K}_{11}$ of the LC Mixtures. 


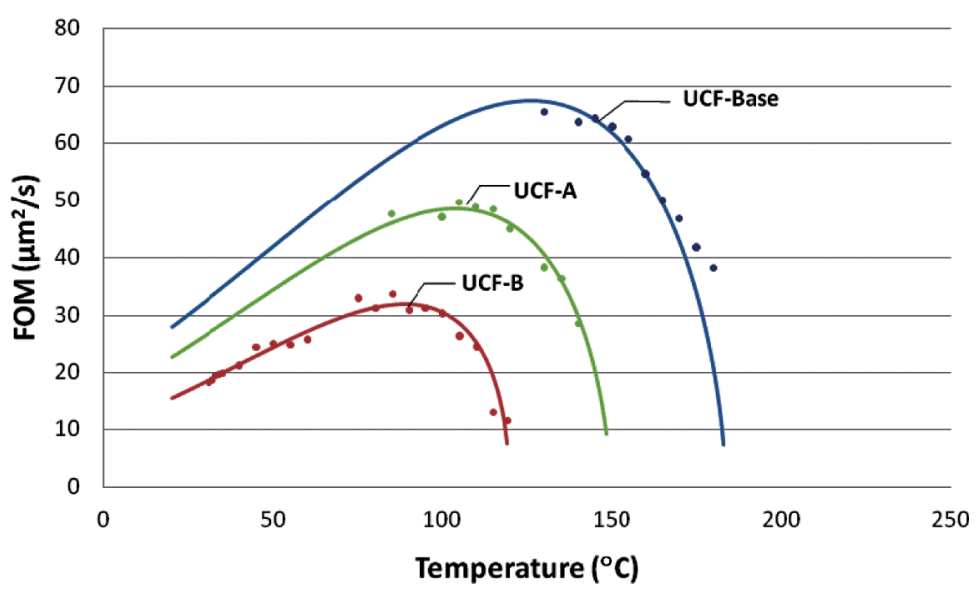

FIGURE 11 Temperature dependent FoM of the LC Mixtures.

presented results, increasing the concentration of the guest mixture improves mesomorphic properties but adversely affects the electrooptic properties due primarily to the lower clearing temperature.

\section{CONCLUSION}

By increasing the number of laterally substituted fluorine, we noted that birefringence gradually decreased with a maximum of $25 \%$ loss if 4-propyl-3", $5^{\prime \prime}$-difluoro- $4^{\prime \prime}$-isothiocyanato-[1,1'; $\left.4^{\prime}, 1^{\prime \prime}\right]$ terphenyl (Comp. 5) was compared with 4-pentyl- $2^{\prime}, 3^{\prime}, 3^{\prime \prime}, 5^{\prime \prime}$-tetrafluoro- $4^{\prime \prime}$ isothiocyanato-[1, $\left.1^{\prime} ; 4^{\prime}, 1^{\prime \prime}\right]$ terphenyl (Comp.1) at the temperature $25^{\circ} \mathrm{C}$. The visco-elastic coefficient was also significantly affected by increasing the number of lateral substituents. Using the same compounds for comparison ( $\mathbf{1}$ and $\mathbf{5}$ ), the $\gamma_{1} / \mathrm{K}_{11}$ coefficient showed a difference of almost $500 \%$ at $25^{\circ} \mathrm{C}$. Therefore, the performance parameter, which is considered as a figure-of-merit, was substantially different for presented compounds. Detailed DSC analysis does not show a significantly lower melting point temperature when the number of fluorine substitutions was increased from two to four. These results led to the conclusion that for the case of rigid terphenyl-NCS core, further fluorination does not affect mesomorphic properties to the same extent as fluorine substitutions in the positions closest to the polar group. The difluorinated NCS terphenyl structure showed the best overall performance of the three fluorinated structures studied. These two compounds will yield faster response times, as the higher 
birefringence value will allow for a thinner cell gap and the lower viscosity will allow the molecules to be rotated faster. The mesomorphic properties (wide nematic range, suppressed smectic phase) and relatively low melting heat enthalpy makes the double-fluorinated NCS terphenyl structure an ideal candidate for eutectic mixtures and potentially an all-isothiocyanatoterphenyl based mixture.

Designing a multi-fluorinated NCS terphenyl host mixture and doping with fluorinated NCS tolanes produced high birefringence mixtures with operating temperatures well below those obtained for single compounds. Future work should concentrate on designing guest mixtures that achieve the best balance between improving mesomorphic properties and retaining the advantageous electro-optic properties of the all-terphenyl base mixture.

\section{REFERENCES}

[1] Efron, U., Wu, S. T., Grinberg, J., \& Hess, L. D. (1985). Visible-to-Infrared dynamic image converter. Opt. Eng., 24, 111-116.

[2] Wu, S. T. (1987). Infrared properties of liquid crystals: an overview. Opt. Eng., 26, $120-128$.

[3] McManamon, P. F., Dorschner, T. A., Corkum, D. L., Friedman, L., Hobbs, D. S., Holz, M., Liberman, S., Nguyen, H. Q., Resler, D. P., Sharp, R. C., \& Watson, E. A. (February 1996). Optical phased array technology. Proc. IEEE, 84, 268-298.

[4] Harriman, J., Gauza, S., Wu, S. T., Wick, D., Bagwell, B., Martinez, T., D. Payne \& Serati, S. (January 2006). Transmissive spatial light modulators with high figureof-merit liquid crystals for foveated imaging applications. Proc. SPIE, 6135, 61350C 1-13.

[5] Wu, S. T. \& Efron, U. (March 1986). Optical properties of thin nematic liquid crystal cells. Appl. Phys. Lett., 48, 624-626.

[6] Gauza, S., Zhu, X., Wu, S. T., Piecek, W., \& Dabrowski, R. (2007). J. Display Technology, 3, 250-252.

[7] Jiao, M., Ge, Z., Song, Q., \& Wu, S. T. (2008). Appl. Phys. Lett., 92, 061102.

[8] Wu, S. T., Margerum, J. D., Meng, H. B., Dalton, L. R., Hsu, C. S., \& Lung, S. H. (August 1992). Room-temperature diphenyl-diacetylene liquid crystals. Appl. Phys. Lett., 61, 630-632.

[9] Sekine, C., Fujisawa, K., Iwakura, K., \& Minai, M. (July 2000). High birefringence phenylacetylene liquid crystals with low viscosity. Mol. Cryst. Liq. Cryst., 364, $711-718$.

[10] Gauza, S., Wen, C. H., Wu, S. T., Janarthanan, N., \& Hsu, C. S. (November 2004). Super high birefringence isothiocyanato biphenyl-bistolane liquid crystals. Jpn. J. Appl. Phys., 43, 7634-7638.

[11] Gauza, S., Wang, H., Wen, C. H., Wu, S. T., Seed, A., \& Dabrowski, R. (June 2003). High birefringence isothiocyanato tolane liquid crystals. Jpn. J. Appl. Phys., Part I, $42,3463-3466$.

[12] Catanescu, C. O. \& Chien, L. C. (January 2006). High birefringence difluoroisothiocyanate biphenyl tolane liquid crystals. Liq. Cryst., 33, 115-120.

[13] Wu, S. T., Hsu, C. S., \& Shyu, K. F. (January 1999). High birefringence and wide nematic range bis-tolane liquid crystals. Appl. Phys. Lett., 74, 344-346. 
[14] Spadlo, A., Dabrowski, R., Filipowicz, M., Stolarz, Z., Przedmojski, J., Gauza, S., Fan, Y. H., \& Wu, S. T. (February 2003). Synthesis, mesomorphic and optical properties of isothiocyanatotolanes. Liq. Cryst., 30, 191-198.

[15] Lin, P. T., Wu, S. T., Chang, C. Y., \& Hsu, C. S. (May 2004). UV stability of high birefringence liquid crystals. Mol. Cryst. Liq. Cryst., 411, 1285-1295.

[16] Wu, S. T., Finkenzeller, U., \& Reiffenrath, V. (June 1989). Physical properties of diphenyldiacetylenicliquid crystals. J. Appl. Phys., 65, 4372-4376.

[17] Gauza, S., Wen, C. H., Tan, B. J., \& Wu, S. T. (October 2004). UV stable high birefringence liquid crystals. Jpn. J. Appl. Phys., 43, 7176-1780.

[18] Huh, I. K. \& Kim, Y. B. (November 2002). Fluoro-isothiocyanated liquid crystal materials with high dielectric anisotropy and voltage holding ratio. Jpn. J. Appl. Phys., 41, 6466-6470.

[19] Malecki, J. A. \& Nowak, J. (May 1999). Intermolecular interactions in benzene solutions of 4-heptyl-3'-cyano-biphenyl studied with non-linear dielectric effects. J. Molecular Liquids, 81, 245-252.

[20] Wu, S. T., Efron, U., \& Hess, L. D. (November 1984). Birefringence measurements of liquid crystals. Appl. Opt., 23, 3911-3915.

[21] Wu, S. T. \& Wu, C. S. (August 1991). Experimental confirmation of Osipov-Terentjev theory on the viscosity of liquid crystals. Phys. Rev. A, 42, 2219-2227.

[22] Wu, S. T., Lackner, A. M., \& Efron, U. (August 1987). Optimal operation temperature of liquid crystal modulators. Appl. Opt., 26, 3441-3445.

[23] Gauza, S., Li, J., Wu, S. T., Spadlo, A., Dabrowski, R., Tzeng, Y. N., \& Cheng, K. L. (August 2005). High birefringence and high resistivity isothiocyanate-based nematic liquid crystal mixtures. Liq. Cryst., 32, 1077-1085.

[24] Gauza, S., Wen, C. H., Wu, B., \& Wu, S. T. (June 2006). High figure-of-merit nematic mixtures based on totally unsaturated isothiocyanate liquid crystals. Liq. Cryst. 33, 705-710. 